\title{
EVALUACIÓN SISTEMÁTICA DEL DESEMPEÑO TÉRMICO DE UN MÓDULO EXPERIMENTAL DE VIVIENDA ALTO ANDINA PARA LOGRAR EL CONFORT TÉRMICO CON ENERGÍA SOLAR
}

\section{SYSTEMATIC EVALUATION OF THE THERMAL PERFORMANCE OF AN EXPERIMENTAL MODULE OF HIGH ANDEAN HOUSING TO ACHIEVE THERMAL COMFORT WITH SOLAR}

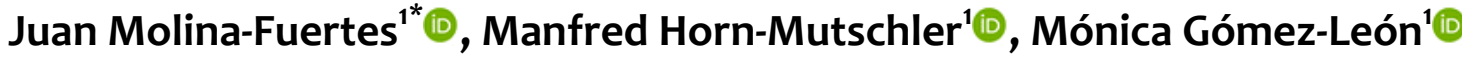

${ }^{1}$ Facultad de Ciencias, Universidad Nacional de Ingeniería, Lima, Perú

Recibido (Recieved): 17/02/2020 Aceptado (Accepted): 05/03/2020

\begin{abstract}
RESUMEN
El objetivo del presente trabajo es evaluar experimentalmente el desempeño térmico de un Módulo Experimental de Vivienda (MEV), construido en la región Alto Andina, mediante la evaluación sistemática de doce configuraciones de operatividad diferentes, desde el manejo nocturno de contraventanas hasta actividad humana, uso de cocinas a gas y uso de dos sistemas de calefacción solar activos. Este objetivo se fundamenta en las mediciones de temperatura y humedad, considerando la primera como el parámetro principal de análisis térmico para verificar el desempeño térmico del MEV mediante la variación de la temperatura interior respecto al exterior, según la configuración del MEV dado a que la variación entre la temperatura diaria promedio interior y exterior muestra la eficacia de las estrategias de calentamiento o enfriamiento. En base a ello, los incrementos de temperatura en el interior del MEV, considerando una operatividad concienzuda de sus técnicas bioclimáticas implementadas, son del orden de $9,5{ }^{\circ} \mathrm{C}$ en promedio $\mathrm{y}$, de hasta $16,6{ }^{\circ} \mathrm{C}$ en horas críticas de la madrugada respecto a la temperatura exterior de $-7,1^{\circ} \mathrm{C}$ a las 6 a.m.

El estudio se complementa con simulaciones térmicas dinámicas utilizando el software EnergyPlus apoyado del entorno gráfico en 3D SketchUp, e interfaz OpenStudio para determinar la temperatura interior y validarlo con la temperatura interior real medida. Los resultados muestran un buen acuerdo entre la simulación y los datos experimentales, con un error cuadrático medio de 15 y $16 \%$ en los ambientes del MEV.
\end{abstract}

Palabras Clave: energía solar, confort térmico, SketchUp, OpenStudio, EnergyPlus.

\section{ABSTRACT}

The objective of this work is to evaluate experimentally the thermal performance of an Experimental Housing Module (MEV) through systematic evaluation of twelve different configurations of operation, from nightly closing of window shutters until overnight stay, human activity, use of gas stoves and use of two active solar heating systems. This objective was based on the measurements of temperature and humidity, considering the first as the main parameter of thermal analysis to verify the thermal performance of the MEV by increasing the internal temperature with respect to the outside, according to the operability of the MEV, given that the variation between the average daily indoor and outdoor temperature, shows the effectiveness of passive heating or cooling strategies. Based on this, the temperature increases inside the MEV considering a conscientious operation of its implemented bioclimatic techniques and internal gains to reach its thermal comfort, are of the order of $9.5^{\circ} \mathrm{C}$ on average and, up to $16.6^{\circ} \mathrm{C}$ during critical hours of the morning with respect to the outside temperature of 7.1 ${ }^{\circ} \mathrm{C}$ at 6 a.m.

The study is complemented by dynamic thermal simulations using the EnergyPlus software supported by the 3D SketchUp graphic environment, and OpenStudio interface to determine the indoor temperature and validate it with the actual measured indoor temperature. The results show a good agreement between the simulation and the experimental data, with an average square error of 15 and $16 \%$ in the MEV environments.

Keywords: solar energy, thermal comfort, SketchUp, OpenStudio, EnergyPlus.

\footnotetext{
${ }^{*}$ Corresponding author.:

E-mail: juan.molina.f@uni.pe
} 


\section{INTRODUCCIÓN}

Hasta hoy en día año tras año entre los meses de junio a agosto principalmente, son noticias de cada día las consecuencias que causa el fenómeno climatológico de las bajas temperaturas o "helada" (descensos de temperatura por debajo o igual a $0{ }^{\circ} \mathrm{C}$ ), en las diferentes zonas Alto Andinas del país a más de $3200 \mathrm{msnm}$, y por encima de los $4200 \mathrm{msnm}$ a $-18^{\circ} \mathrm{C}[1]$. Estas condiciones climáticas al sobrepasar los umbrales de adaptación de la población pueden ocasionar daños a la vida, salud, educación, actividad agrícola y ganadera e infraestructura, principalmente a las poblaciones en situación de alta vulnerabilidad, sea por su condición social (pobreza y pobreza extrema), por su edad (niños y adultos mayores), y ubicación territorial [2]. Hasta fines de junio de 2016, el Instituto de Defensa Civil (INDECI), informó que las bajas temperaturas habían afectado la salud de 257.251 personas ( $51 \%$ en Puno y $21 \%$ en Cusco). El mismo reporte señala que 48 niños menores de 5 años murieron por causas relacionadas con el frío entre mediados de abril y el 20 de junio [3].

Ante esta situación y para paliar estas consecuencias, una alternativa es que las poblaciones cuenten con viviendas dignas donde la energía solar sea considerada de manera obligatoria en la etapa de diseño debido a que en las zonas Alto Andinas y principalmente al Sur del país, la radiación solar bordea anualmente los $6 \mathrm{kWh} /\left(\mathrm{m}^{2} \bullet\right.$ día $)[4,5]$.

Así mismo, las instituciones de diversas índoles sean privadas, estatales y ONGs, tienen que realizar la transferencia de tecnología en base a estudios y experiencias realizados sobre el clima, materiales locales, construcciones bioclimáticas, y confort térmico. Materiales locales como el adobe por ejemplo cuyos conocimientos ancestrales de buenas prácticas constructivas se han perdido al pasar los años y tienen que recuperarse añadiendo estudios y técnicas modernas en su fabricación. Además, del conocimiento de sus propiedades térmicas [6] que se emplean como variable de entrada a los softwares de simulación dinámica para determinar el rendimiento térmico/energético de edificaciones [7], y evitar posibles incertidumbres al tomar valores de referencias internacionales.

En este contexto, el presente estudio tiene como objetivo evaluar el desempeño térmico de un módulo experimental de vivienda alto andina en base a doce configuraciones de medida con data meteorológica registrada in situ, data de un año meteorológico típico, y data como resultado del empleo de software de simulación EnergyPlus previamente validada.

\section{ANTECEDENTES}

En el ámbito local desde hace una década, se ha intensificado el estudio del confort térmico en zonas Alto Andinas para mitigar el fenómeno climatológico de las bajas temperaturas o "helada", principalmente mediante la ejecución de proyectos de tesis de investigación de título profesional y grado de maestría $[8,9]$. Tal es así que la UNI desde el 2009 por medio de la Facultad de Ciencias y el CER-UNI, ha enfatizado el estudio experimental in situ del confort térmico en poblaciones rurales por encima de los $3000 \mathrm{msnm}$, en base a construcciones de módulos habitables de vivienda partiendo de un diagnóstico y diseño bioclimático acorde a dichas poblaciones respetando las costumbres y re-valorando las técnicas constructivas tradicionales [10]; priorizando el uso del recurso solar en abundancia por estas zonas en especial las Alto Andinas, buen aislamiento, hermeticidad, adecuada operatividad de los elementos constructivos sobre la envolvente, e implementación de sistemas de climatización pasiva o activa con energía solar [11]. Los estudios realizados contemplan desde la etapa de diseño, el uso de programas de modelado en 3D y simulación computacional como el EnergyPlus, SketchUp, y OpenStudio.

En un contexto similar pero más enfocado a la construcción bioclimática a través de proyectos pilotos en zonas Alto Andinas, entidades del estado como el Ministerio de Vivienda Construcción y Saneamiento (MVCS) en colaboración con universidades y ONGs, publicó el 2014 el libro "Abrigando Hogares" [12], donde recopila una serie de técnicas de aislamiento sobre la envolvente de viviendas. La misma entidad el mismo año publica la Norma Técnica Peruana EM.110 "Confort Térmico y Lumínico con Eficiencia Energética" [13], aún de aplicación voluntaria para nuevas construcciones o rehabilitaciones de viviendas donde dan hincapié, para nueve zonas bioclimáticas, el cumplimiento de valores de transmitancia térmica (U) en muros, techo y piso. Con estas exigencias, la norma EM.100 solo prevé el nivel de aislamiento de los materiales (cálculo de $U$ conociendo su conductividad térmica y espesor) obviando su capacidad calorífica como material acumulador de calor (inercia térmica), parámetro físico de importancia donde se emplea materiales de construcción de alta masa térmica como el adobe o tapial y, donde el clima se presenta con altas fluctuaciones de temperatura y abundante recurso solar. En base a ello, la norma solo considera cálculos en estado estacionario como la gran mayoría de normas de Sudamérica [14].

Otras instituciones que han contribuido al estudio de las construcciones bioclimáticas enfocados a su mejora térmica son las ONGs como Soluciones Prácticas (antes ITDG), que ha elaborado el libro "Calefacción solar para regiones frías", donde, se 
presenta el aprovechamiento del recurso solar para el diseño de ambientes interiores no solo para el bienestar humano sino también para la ganadería y agricultura [15]. Otra ONG es Care Perú que a partir de cinco modelos de vivienda construidos en la provincia de Castrovirreyna en Huancavelica, recomiendan en base a medidas experimentales y empleo de simulaciones dinámicas, el uso de claraboyas en el techo e invernadero adosado [16].

A nivel internacional los estudios respecto al diseño bioclimático y específicamente al confort térmico son amplios, como el desarrollado por Agugliaro [17] que recopila información de diversas estrategias y técnicas constructivas en base al clima empleando la carta psicrométrica de Givoni. Otros por ejemplo resaltan los diferentes métodos y técnicas que se han empleado en todo el mundo para lograr reformas de rendimiento y adaptar los edificios a los estándares actuales de eficiencia energética y confort térmico es esencial para mejorar la sostenibilidad y el rendimiento energético [18]. Así como también resaltan que el uso de un software dinámico es más apropiado para tratar las propiedades inerciales de la estructura para calcular de manera más detallada las cargas térmicas anuales y obtener más información sobre las zonas de calentamiento individuales [19].

En este contexto, el presente trabajo se refiere al estudio del confort térmico mediante evaluaciones sistemáticas de un módulo experimental de vivienda (MEV), complementado con la modelación y simulación dinámica empleando el software EnergyPlus [20], y su posterior validación. El MEV se construyó y evaluó en forma preliminar por el MSc. Rafael Leonardo Espinoza Paredes [21]. El MEV se encuentra en San Francisco de Raymina, una comunidad al sur de Ayacucho a $3700 \mathrm{msnm}$. El presente estudio comprendió doce configuraciones de medida del comportamiento del MEV, como etapa previa la rehabilitación del MEV y después como etapa inicial la evaluación de su desempeño térmico usando solamente técnicas bioclimáticas arquitectónicas pasivas de aislamiento térmico: piso aislado, dobles puertas de madera, ventanas con doble vidrio $y$ coberturas de madera, y techo con cielo raso. Una segunda configuración de medida incluyó adicionar a la evaluación anterior, a dos sistemas de calefacción solar, los denominados "muro radiante" y "tubo radiante", que utilizan como fuente de energía el Sol y el agua como medio de acumulación de calor en forma de calor sensible. Y la última configuración consistió en la evaluación con técnicas de aislamiento, sistemas de calefacción, la inclusión de cargas internas, pernoctación, y ocupación humana.

\subsection{ESPECIFICACIONES DEL MÓDULO EXPERIMENTAL DE VIVIENDA}

El MEV se ubica en la comunidad de San Francisco de Raymina a 3700 msnm (latitud 13 $45^{\prime} 40^{\prime \prime}$ 's y longitud $73^{\circ} 51^{\prime} 26^{\prime \prime}$ ), en el distrito de Huambalpa, provincia Vilcashuamán, región Ayacucho, al sur del Perú. Se asienta sobre un área de $27,36 \mathrm{~m}^{2}$ distribuidos en dos ambientes independientes de base cuadrada de $3 \mathrm{~m} \times 3$ $\mathrm{m}$, y altura $2,2 \mathrm{~m}$ entre techo y cielo raso, separados por un muro divisorio de adobe, y un espacio entre el techo y cielo raso tipo ático. El área superficial exterior de la envolvente es de $113,24 \mathrm{~m}^{2}$, y el volumen total del aire interior de $56 \mathrm{~m}^{3}$ (39,6 $\mathrm{m}^{3}$ de los ambientes interiores, y $16.4 \mathrm{~m}^{3}$ del ático). Durante el periodo de evaluación en la configuración con pernoctación, la casa fue habitada por dos adultos y un adolescente. El techo es a dos aguas de chapa metálica, las paredes de adobes de 0,4 $\mathrm{m} \times 0,4 \mathrm{~m} \times 0,1 \mathrm{~m}$ recubiertos por el exterior con una capa de barro de 0,02 m, una vista del MEV se muestra en la Fig. 1a. Las ventanas que son dos por ambiente, se orientan una al este, y la otra al oeste, ambos con hojas que se abren, marcos de madera y doble vidrio espaciados 0,01 m, todas llevan como protección térmica interior, un panel de madera sólida de 0,02 $\mathrm{m}$ de espesor que hace de contraventana y se coloca manualmente al anochecer (18 p.m.), y se retira al día siguiente por las mañanas (6 a.m.).

Cada ambiente cuenta con doble puerta espaciadas $0,65 \mathrm{~m}$ al interior tipo esclusa, orientadas al norte y sur. El piso a partir de tierra apisonada y nivelada, vista de abajo hacia arriba se compone de: polietileno, entramado de madera con espacios de aire de 0,05 m de altura, y paneles tipo sándwich de chapa metálica por los extremos con 0,05 $\mathrm{m}$ de poliuretano expandido en su centro. Del mismo material tipo sándwich es el cielo raso. Cada ambiente tiene instalado en su interior un sistema de calefacción solar activo denominado tubo radiante (ambiente norte, Fig. 1b), y pared radiante (ambiente sur, Fig. 1c), ambos emplean agua como fluido caloportador y acumulador de energía por calor sensible. El tubo radiante se opera manualmente a través de una serie de válvulas de paso donde, a las 18 p.m. se deposita en su interior 65 litros de agua caliente proveniente de un colector solar tipo caja manteniéndose ahí hasta el día siguiente a las 6 a.m., y que posteriormente es conducido hacia un tanque enterrado de 95 litros y devuelto al colector solar mediante una bomba de $30 \mathrm{~W}$ accionada por un panel solar de $55 \mathrm{~W}_{\mathrm{p}}$, repitiéndose el proceso del ciclo cerrado del agua diariamente. Por el lado del sistema de calefacción de pared radiante, este es automático, el agua calentada en un colector solar tipo piscina fluye hacia un serpentín adosado en la pared divisoria del ambiente sur cuando, la diferencia entre la 
temperatura superficial a la salida del agua caliente del colector solar con la temperatura del aire interior del ambiente sur, sea igual o mayor a $5^{\circ} \mathrm{C}$.

\section{METODOLOGÍA}

De acuerdo a los objetivos del presente estudio, se evalúa el MEV de manera sistemática a partir de doce configuraciones de medida, para determinar su desempeño térmico. Configuraciones que van con el MEV cerrado y abierto las 24 horas solo con técnicas de aislamiento pasivo (TAAP); operatividad de puertas, ventanas, contrapuertas (CP), y contraventanas (CV), inclusión de dos sistemas activos de calefacción solar (SC), cocina a gas (CG) como carga interna, y pernoctación $(P)$, las cuales se describen en la tabla I de acuerdo a los tiempos y fechas de evaluación entre los años 2016 a 2017, periodo de tiempo donde se registró in situ, data meteorológica del lugar para posteriormente, generar un archivo climático con extensión EPW (EnergyPlus Weather) de entrada al EnergyPlus a través del software Meteonorm.

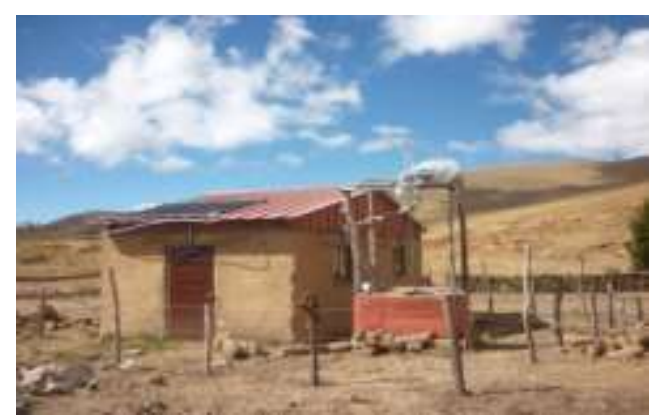

(a)

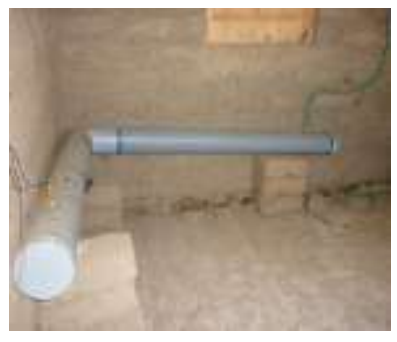

(b)

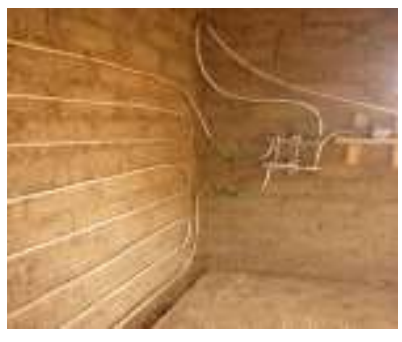

(c)
Fig. 1. Vistas del módulo y sistemas de calefacción solar. (a) MEV, (b) tubo radiante en el ambiente norte, y (c) serpentín del sistema muro radiante en el ambiente sur y adosado a la pared divisoria.

TABLA

Configuraciones de medidas en el MEV

\begin{tabular}{|c|c|c|}
\hline & Configuraciones de medidas & Fecha \\
\hline 1 & $\begin{array}{l}\text { MEV Sólo con técnicas arquitectónicas } \\
\text { de aislamiento pasivo (TAAP) y manejo } \\
\text { de contraventanas (CV). }\end{array}$ & $\begin{array}{l}05-29 \\
\text { Jul. }\end{array}$ \\
\hline 2 & $\begin{array}{l}\text { MEV con TAAP, manejo de CV y, adición } \\
\text { de sistemas de calefacción solar activos } \\
\text { (SC). }\end{array}$ & $\begin{array}{l}24 \text { Ago. - } \\
26 \text { Sep. }\end{array}$ \\
\hline 3 & $\begin{array}{l}\text { MEV con ambientes cerrados las 24h: } \\
\text { con TAAP, sin SC, con CV y }\end{array}$ & $\begin{array}{l}27 \text { Sep. - } \\
11 \text { Oct. }\end{array}$ \\
\hline
\end{tabular}

\begin{tabular}{|c|c|c|}
\hline & contrapuertas (CP). & \\
\hline 4 & $\begin{array}{l}\text { MEV con TAAP, } \sin C V, \sin C P, \text { y } \sin S C \\
\text { las } 24 \mathrm{~h} \text { : Ambiente Sur }(S) \text { y Norte }(N) .\end{array}$ & $\begin{array}{l}12-24 \\
\text { Oct. }\end{array}$ \\
\hline 5 & $\begin{array}{l}\text { MEV con TAAP, manejo de } \mathrm{CV} \text { y con } \mathrm{SC} \\
\text { ambiente } \mathrm{S} \text { y } \mathrm{N} \text {; Ambiente } \mathrm{S} \text { con cocina } \\
\text { a gas (CG). }\end{array}$ & $\begin{array}{l}25 \text { Oct. - } \\
6 \text { Nov. }\end{array}$ \\
\hline 6 & $\begin{array}{l}\text { MEV con TAAP, manejo de } \mathrm{CV} \text {, y con } \mathrm{SC} \\
\text { ambiente } \mathrm{S} \text { y } \mathrm{N} \text {; Ambiente } \mathrm{N} \text { con } \\
\text { cocinas a gas. }\end{array}$ & $\begin{array}{l}7 \text { Nov. - } \\
4 \text { Dic. }\end{array}$ \\
\hline 7 & $\begin{array}{l}\text { MEV con TAAP, manejo de CV, con SC y } \\
\text { con CG ambiente S y N. }\end{array}$ & $\begin{array}{l}5-12 \\
\text { Dic. }\end{array}$ \\
\hline 8 & $\begin{array}{l}\text { MEV con TAAP, manejo de CV, con SC y } \\
\text { con CG ambiente S y N; Pernoctación } \\
\text { (P) ambiente S. }\end{array}$ & $\begin{array}{l}13-20 \\
\text { Dic. }\end{array}$ \\
\hline 9 & $\begin{array}{l}\text { MEV con TAAP, manejo de CV, con SC y } \\
\text { con CG ambiente S y N; P y aislamiento } \\
\text { del colector solar (ACS) ambiente S. }\end{array}$ & $\begin{array}{l}21-23 \\
\text { Dic. }\end{array}$ \\
\hline 10 & $\begin{array}{l}\text { MEV con TAAP, sin CV las } 24 \mathrm{~h} \text {, sin CG, } \\
\text { con ACS y sin P ambiente S y N. Sólo } \\
\text { con SC ambiente S. }\end{array}$ & 24 Dic-06 Feb. \\
\hline 11 & $\begin{array}{l}\text { MEV con TAAP, manejo de CV, con SC, } \\
\text { con ACS y con CG ambiente S y N; P y } \\
\text { ocupación humana (OH) por } 5 \text { h } \\
\text { Ambiente S. }\end{array}$ & 07-23 Feb. \\
\hline 12 & $\begin{array}{l}\text { MEV con TAAP, manejo de CV, con SC, } \\
\text { con ACS y CG ambiente S y N; P y OH } \\
\text { por } 5 \text { h Ambiente N. }\end{array}$ & 24-28 Feb. \\
\hline
\end{tabular}

Nota: Cada configuración de medida en la fecha especificada, representa la operatividad del MEV para determinar de acuerdo a cada una de ellas, el incremento de temperatura al interior del MEV.

Así mismo, el estudio se complementa con el modelado y simulación del MEV empleando el software EnergyPlus para determinar la temperatura interior, que se contrasta con la temperatura interior real medida para la validación del modelo y analizar el desempeño térmico del MEV. La Fig. 2 muestra el diagrama de flujo del proceso de modelado y simulación en EnergyPlus, el cual se asocia de otros dos programas como el SketchUp, y Legacy OpenStudio [22, 23], con el primero se genera la geometría en 3D del edificio, y con el segundo, se genera las zonas térmicas (volúmenes de aire a temperaturas uniformes) de acuerdo a los espacios o volúmenes (masas) de aire a analizar. EnergyPlus es el motor de cálculo térmico/energético que considera los flujos de calor dependientes del tiempo, es decir, en estado transitorio. Dado a que entre las variables principales de entrada a la simulación están las propiedades termofísicas y ópticas de los materiales, las primeras se tomaron de la Norma Técnica Peruana EM.110 y las segundas de acuerdo a la recomendación del programa, 0,7 para la absortancia solar, y 0,9 para la absortancia térmica. Así mismo, los coeficientes de transferencia de calor por convección se consideraron también de la norma EM.110, para el piso interior $\mathrm{y}$ exterior $11,1 \mathrm{~W} / \mathrm{m}^{2} \cdot{ }^{\circ} \mathrm{C}$, para el techo interior y exterior 11,1 y $20 \mathrm{~W} / \mathrm{m}^{2} \cdot{ }^{\circ} \mathrm{C}$, y para las paredes interior y exterior 9 y $16,6 \mathrm{~W} / \mathrm{m}^{2} \cdot{ }^{\circ} \mathrm{C}$.

Las simulaciones del MEV se consideran para la configuración de medida 12, del 24 al 28 de febrero del 2017, donde se tiene en operatividad ambos ambientes 
diferenciando solo uno del otro en la pernoctación y operatividad humana por $5 \mathrm{~h}$ que se da en el ambiente norte.

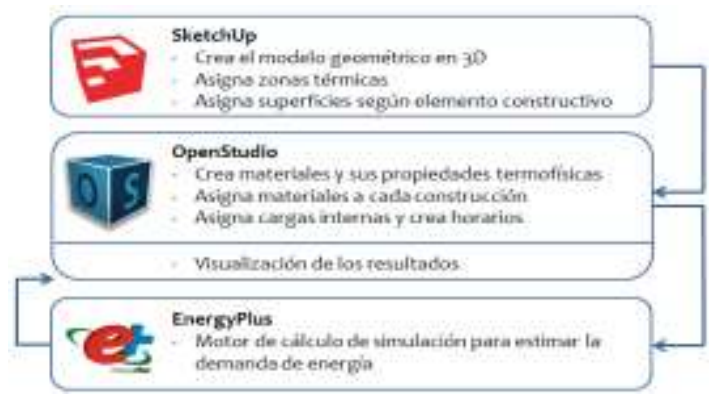

Fig. 2. Diagrama de flujo del proceso de simulación EnergyPlus. Adaptado de [24].

\subsection{CONFIGURACIÓN EXPERIMENTAL Y MODELADO CON ENERGYPLUS}

Los ambientes norte y sur del MEV para cada configuración de medida de acuerdo a sus tiempos de evaluación, se monitorearon térmicamente empleando sensores de temperatura Pt10o (Fig. 3a), conectados a registradores de datos de marca ONSET HOBO U12-006 (Fig. 3b) de cuatro canales programados cada 30 minutos. La medida de la humedad relativa se realizó con registradores de datos de la misma marca pero modelo U12-012 que cuentan con sensores internos y que además miden, luminosidad, temperatura, y cuentan con un canal externo para medir temperatura. Las medidas de los parámetros meteorológicos externos como temperatura del aire, humedad relativa, radiación solar global sobre superficie horizontal, velocidad y dirección del viento, fueron realizados empleando una estación meteorológica HOBO (Fig. 3C) ubicada a $100 \mathrm{~m}$ del MEV. Las medidas se realizaron desde el 5 de julio del 2016 al 28 de febrero del 2017.

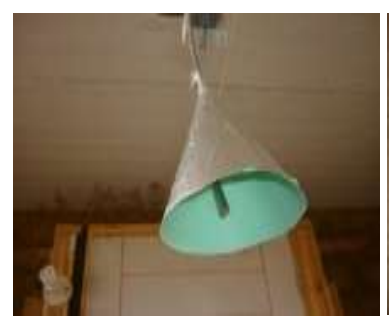

(a)

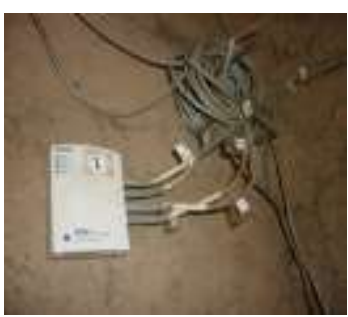

(b)

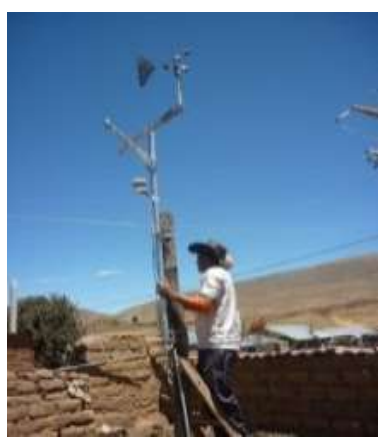

(c)

Fig. 3. Instrumentos de medición. (a) Sensor Pt10o con protección de la radiación solar, (b) registrador de datos de temperatura de cuatro canales, y (c) estación meteorológica.

La simulación de la presente investigación se realiza empleando el programa informático Energyplus de uso mundial y propiedad del Departamento de Energía de los Estados Unidos, es de código abierto y uso gratuito al igual que sus complementos SketchUp y Legacy OpenStudio, el primero de propiedad de Trimble Navigation, y el segundo del Laboratorio Nacional de Energías Renovables de los Estados Unidos (NREL). EnergyPlus permite determinar las demandas de energía de calefacción y refrigeración en un edificio así como, la temperatura interior y otras variables entre ellas solo por nombrar, la energía por radiación solar incidente en las superficies exteriores e interiores, las temperaturas superficiales exteriores e interiores, el promedio de la transferencia de energía por conducción desde las superficies, y el almacenamiento de energía en superficies. Todo ello a partir del ingreso de variables como el archivo de clima y diseño geométrico en 3D del edificio, los algoritmos de cálculos de los coeficientes de convección interior y exterior, el algoritmo del balance de calor, el paso de tiempo en la solución de las ecuaciones y sus cálculos, información del edificio, el control de la simulación, ubicación geográfica del edificio, temperatura del terreno, materiales opacos y transparentes con sus propiedades termofísicas y ópticas, configuraciones constructivas, entre otras.

El modelado del MEV se realiza considerando tres zonas térmicas de las cuales para el estudio se considera solo dos (Fig. 4a), la zona térmica denominada ambiente sur (Fig. 4b), y ambiente norte (Fig. 4c), debido a que nos interesa evaluar el desempeño térmico de dichos ambientes. 


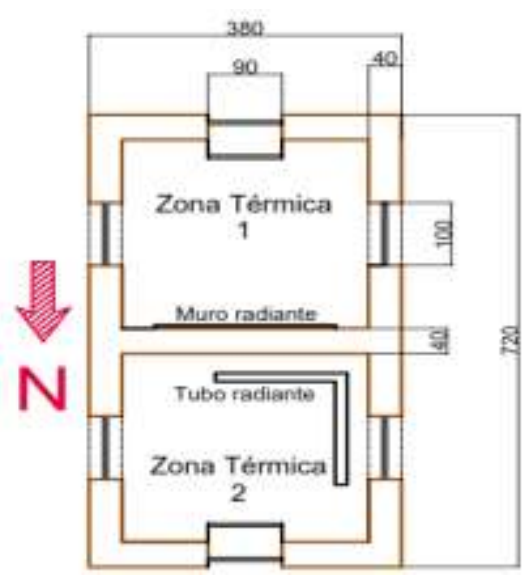

(a)

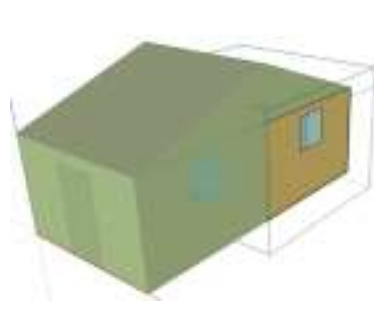

(b)

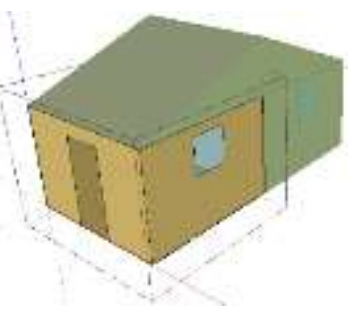

(c)
Fig. 4. Representación de las zonas térmicas. (a) Esquema de las zonas, (b) ambiente sur, y (c) ambiente norte.

\section{ANÁLISIS DE RESULTADOS}

Se presentan resultados y análisis de los datos experimentales registrados y simulaciones en el MEV a través de la medición de variables ambientales interiores y exteriores como la temperatura del aire, humedad relativa y radiación solar. La monitorización de estas variables será útil para comprender el comportamiento térmico del MEV desde el punto de vista energético en lo que refiere a ganancias $y$ pérdidas de energía a través de su envolvente, cargas internas, y actividad humana; a la vez, que servirá de referencia para otras investigaciones que involucren el comportamiento térmico de viviendas.

\subsection{CONDICIONES CLIMÁTICAS EXTERIORES}

Del tratamiento de datos registrados en San Francisco de Raymina durante la etapa de medida del 5 de julio del 2016 al 28 de febrero del 2017, el promedio de temperatura exterior oscila entre $2,6{ }^{\circ} \mathrm{C}$ y $16,9{ }^{\circ} \mathrm{C}$ con una temperatura promedio de $9,3{ }^{\circ} \mathrm{C}$, una temperatura mínima horaria que llega hasta $-7,1^{\circ} \mathrm{C}$ a las 6 a.m. el día 29 de agosto del 2016, y máxima horaria de $24,1{ }^{\circ} \mathrm{C}$ alcanzada al medio día el 12 de noviembre del mismo año; la humedad relativa oscila entre $42.3 \%$ y $86,9 \%$ con una humedad relativa promedio de 69,2 \%; una Irradiancia solar promedio diario máximo de 1091,29 $\mathrm{W} / \mathrm{m}^{2}$ con un promedio de $480,68 \mathrm{~W} / \mathrm{m}^{2}$, y energía solar promedio diario de $5,98 \mathrm{kWh} /\left(\mathrm{m}^{2} \cdot\right.$ día $)$.

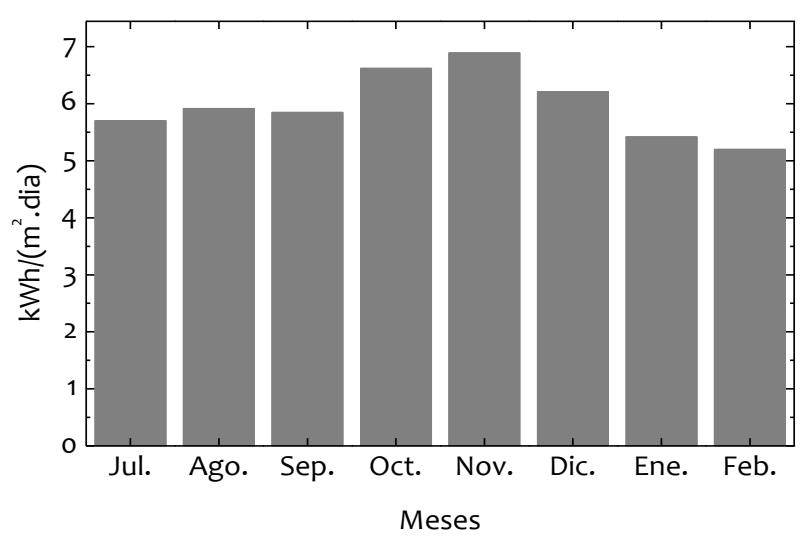

Fig. 5. Energía solar media diaria $\left(k W \mathrm{~h} /\left(m^{2} \cdot d i ́ a\right)\right)$ por mes para San Francisco de Raymina según data registrada del 5 de julio del 2016 al 28 de febrero del 2017.

\subsection{TEMPERATURAS MEDIAS DIARIAS INTERIOR Y EXTERIOR}

La tabla II muestra las configuraciones de medida con los incrementos de temperatura del aire interior (Ti) respecto al exterior (Text) alcanzados en los ambientes del MEV, presentándose un incremento mínimo de $2,7^{\circ} \mathrm{C}$ en la configuración de medida 1 , hasta incrementos de $9,5^{\circ} \mathrm{C}$ en la configuración de medida 12 donde, se considera en ambos ambientes, el aislamiento, manejo de contraventanas, funcionamiento de los sistemas de calefacción solar, aislamiento del colector solar del sistema denominado muro radiante $y$, encendido de la cocina a gas. $Y$ solo en el ambiente sur, pernoctación de dos adultos y un adolescente, y operatividad humana por 5 horas en el ambiente norte.

TABLA II

Incrementos de temperatura media diaria al interior (Ti) del MEV respecto al exterior (Text), ambientes sur (Amb S) y norte (Amb N), según configuraciones de medida.

\begin{tabular}{|c|c|c|c|c|}
\hline \multicolumn{2}{|c|}{ Configuraciones de medida } & \multirow[t]{2}{*}{ Fecha } & \multicolumn{2}{|c|}{$\begin{array}{c}\text { Incrementos de } \\
\text { temperatura } \\
(\mathrm{Ti}-\mathrm{Text})\left({ }^{\circ} \mathrm{C}\right)\end{array}$} \\
\hline & & & $\begin{array}{c}A m b \\
S\end{array}$ & $\begin{array}{c}A m b \\
N\end{array}$ \\
\hline 1 & $\begin{array}{l}\text { MEV Sólo con técnicas } \\
\text { arquitectónicas } \\
\text { aislamiento pasivo (TAAP) } \\
\text { y manejo de } \\
\text { contraventanas (CV). }\end{array}$ & $\begin{array}{l}\text { 5-29 } \\
\text { Jul. }\end{array}$ & 2,7 & 5,3 \\
\hline 2 & $\begin{array}{l}\text { MEV con TAAP, manejo de } \\
\text { CV y, adición de sistemas } \\
\text { de calefacción solar } \\
\text { activos (SC). }\end{array}$ & $\begin{array}{l}24 \text { Ago. } \\
-26 \\
\text { Sep. }\end{array}$ & 4,2 & 5,3 \\
\hline 3 & $\begin{array}{l}\text { MEV con ambientes } \\
\text { cerrados las 24h: con } \\
\text { TAAP, sin SC, con CV y } \\
\text { contrapuertas }(C P) \text {. }\end{array}$ & $\begin{array}{l}27 \text { Sep. } \\
\text { - } 11 \text { Oct. }\end{array}$ & 3,3 & 4,0 \\
\hline 4 & $\begin{array}{l}\text { MEV con TAAP, sin CV, sin } \\
\text { CP, y sin SC las } 24 \mathrm{~h} \text { : } \\
\text { Ambiente Sur (S) y Norte } \\
(\mathrm{N}) \text {. }\end{array}$ & $\begin{array}{l}12-24 \\
\text { Oct. }\end{array}$ & 3,7 & 4,2 \\
\hline 5 & $\begin{array}{l}\text { MEV con TAAP, manejo de } \\
\text { CV y con SC ambiente } \mathrm{S} \text { y } \\
\mathrm{N} \text {; Ambiente } \mathrm{S} \text { con cocina }\end{array}$ & $\begin{array}{c}25 \text { Oct. } \\
-6 \\
\text { Nov. }\end{array}$ & 5,8 & 5,8 \\
\hline
\end{tabular}




\begin{tabular}{|c|c|c|c|c|}
\hline & a gas (CG). & & & \\
\hline 6 & $\begin{array}{l}\text { MEV con TAAP, manejo de } \\
\mathrm{CV}, \text { y con SC ambiente } \mathrm{S} y \\
\mathrm{~N} ; \text { Ambiente N con } \\
\text { cocinas a gas. }\end{array}$ & $\begin{array}{c}7 \text { Nov. - } \\
4 \text { Dic. }\end{array}$ & 4,8 & 5,8 \\
\hline 7 & $\begin{array}{l}\text { MEV con TAAP, manejo de } \\
\mathrm{CV} \text {, con SC y con CG } \\
\text { ambiente Sy N. }\end{array}$ & $\begin{array}{l}\text { 5-12 } \\
\text { Dic. }\end{array}$ & 6,5 & 8,4 \\
\hline 8 & $\begin{array}{l}\text { MEV con TAAP, manejo de } \\
\text { CV, con SC y con CG } \\
\text { ambiente S y } \mathrm{N} \text {; } \\
\text { Pernoctación } \\
\text { ambiente } \mathrm{S} \text {. }\end{array}$ & $\begin{array}{c}13-20 \\
\text { Dic. }\end{array}$ & 8,3 & 8,7 \\
\hline 9 & $\begin{array}{l}\text { MEV con TAAP, manejo de } \\
\text { CV, con SC y con CG } \\
\text { ambiente S y N; P y } \\
\text { aislamiento del colector } \\
\text { solar (ACS) ambiente S. }\end{array}$ & $\begin{array}{c}21-23 \\
\text { Dic. }\end{array}$ & 9,2 & 8,8 \\
\hline 10 & $\begin{array}{l}\text { MEV con TAAP, sin CV las } \\
24 \text { h, sin CG, con ACS y sin } \\
\text { P ambiente S y N. Sólo con } \\
\text { SC ambiente S. }\end{array}$ & $\begin{array}{l}24 \text { Dic. } \\
-6 \\
\text { Feb. }\end{array}$ & 5,2 & 4,8 \\
\hline 11 & $\begin{array}{l}\text { MEV con TAAP, manejo de } \\
\mathrm{CV} \text {, con SC, con ACS y con } \\
\mathrm{CG} \text { ambiente } \mathrm{S} \text { y N; P y } \\
\text { ocupación humana (OH) } \\
\text { por } 5 \text { h Ambiente } \mathrm{S} \text {. }\end{array}$ & $\begin{array}{l}7-23 \\
\text { Feb. }\end{array}$ & 8,9 & 7,6 \\
\hline 12 & $\begin{array}{l}\text { MEV con TAAP, manejo de } \\
\text { CV, con SC, con ACS y CG } \\
\text { ambiente S y N; P y OH por } \\
\text { 5h Ambiente N. }\end{array}$ & $\begin{array}{c}24-28 \\
\text { Feb. }\end{array}$ & 7,7 & 9,5 \\
\hline
\end{tabular}

Nota: Se muestra el incremento de temperatura para cada configuración de medida en cada ambiente del MEV. En la configuración 1 (baja operatividad), el incremento en el ambiente sur es de $2,7{ }^{\circ} \mathrm{C}$, y en el ambiente norte $5,3{ }^{\circ} \mathrm{C}$; y para mayores operatividades como en la configuración 12, el incremento es de 7,7 ${ }^{\circ} \mathrm{C}$ y $9,5^{\circ} \mathrm{C}$ en el ambiente sur y norte.

Cabe resaltar que para el 29 de agosto del 2016 para una temperatura horaria mínima exterior de $-7,1{ }^{\circ} \mathrm{C}$, e interior de $9,5^{\circ} \mathrm{C}$ en el ambiente norte, se presentan el mayor incremento de temperatura de $16,6^{\circ} \mathrm{C}$.

La Fig. 6 muestra el comportamiento de las temperaturas medias diarias interior del ambiente sur (Ti Amb S) y norte (Ti Amb N), con el exterior (Text). Los números en la gráfica entre líneas punteadas de color rojo representan cada configuración de medida. Empleando el modelo adaptativo de Humphreys (1976) se determina que la temperatura de neutralidad o de confort térmico para San Francisco de Raymina es 16,8 ${ }^{\circ} \mathrm{C}$, con un rango de variación de $\pm 3{ }^{\circ} \mathrm{C}$ debido al valor de su oscilación térmica $\left(14,8^{\circ} \mathrm{C}\right)[25]$.

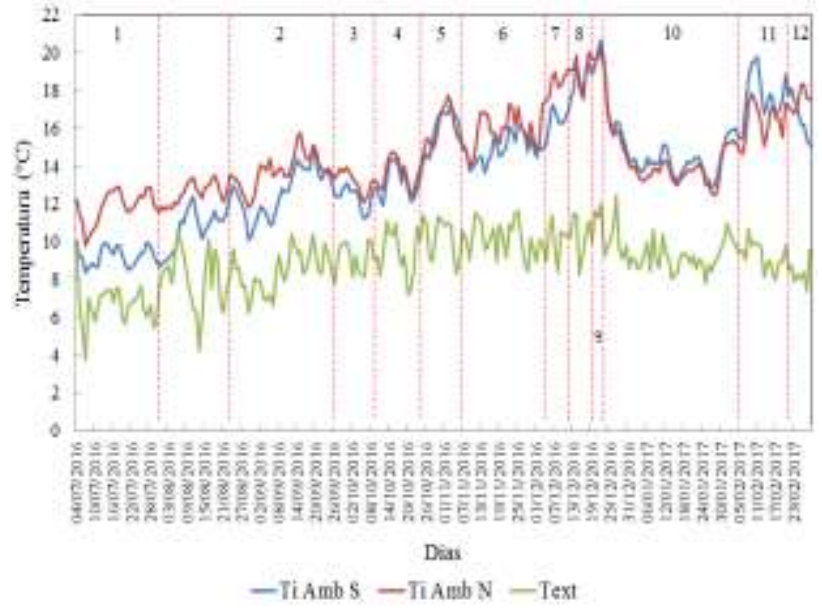

Fig. 6. Temperaturas medias diarias, interior del MEV (Ti) y exterior (Text), para las 12 configuraciones de medida (en números entre líneas verticales punteadas color rojo) en el ambiente Sur (Ti Amb S) y Norte (Ti Amb N).

\subsection{SIMULACIÓN DINÁMICA PARA PREDECIR EL COMPORTAMIENTO TÉRMICO DEL MEV}

Se presente en la Fig. 7 las temperaturas medidas horarias diarias interiores ( $\mathrm{Ti}$ ) y exterior (Text), medidas y simuladas para los ambientes Sur (Fig. 7a), y ambiente Norte (Fig. 7b), para la configuración de medida 12 del 24 al 28 de febrero del 2017. El pico en ambas figuras entre las 19 y 20 p.m., representa el empleo de la cocina a gas en ambos ambientes cuyo encendido se producía a las 19 p.m., y el apagado a las 19:30 p.m. El consumo de gas propano diario en los ambientes fue aproximadamente de $110 \mathrm{~g}$. En una hora el aporte energético de las cocinas al MEV fue en promedio de $1,55 \mathrm{kWh}$. En ambos ambientes la temperatura media mínima esta por los $15{ }^{\circ} \mathrm{C}$ (temperatura que podría considerarse representativa del bienestar térmico alto andino), con una amortiguación térmica de la $\mathrm{Ti}$ respecto a la Text debido a los componentes de su envolvente, baja. La oscilación térmica pasa de $8,8^{\circ} \mathrm{C}$ en el exterior, a $2,2{ }^{\circ} \mathrm{C}$ en el interior, esto debido a la masa térmica de la envolvente del MEV, principalmente de las paredes de adobe cuyo espesor es de $0,4 \mathrm{~m}$. 


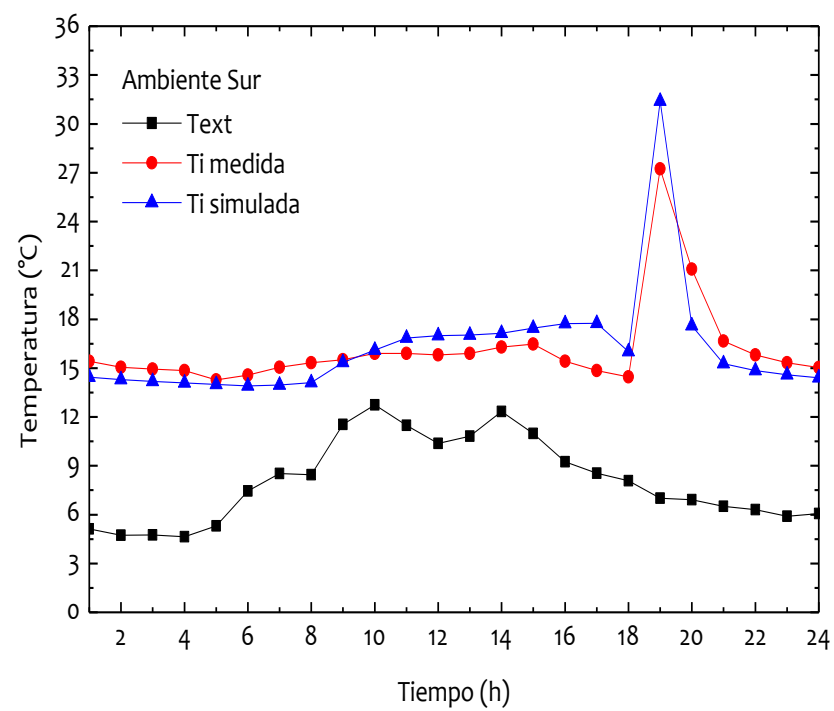

(a)

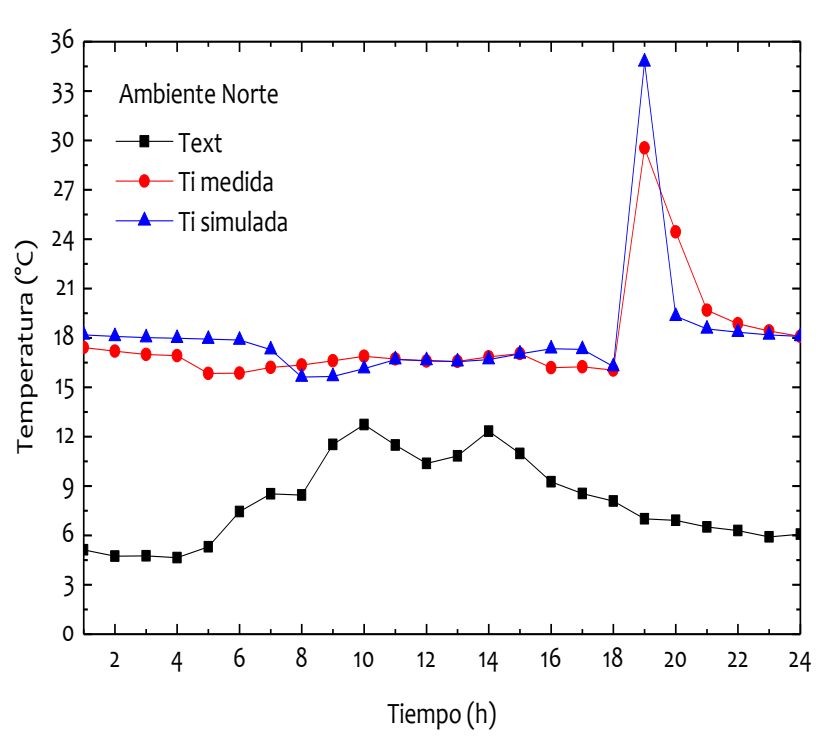

(b)

Fig. 7. Temperaturas medias horarias diarias medidas y simuladas, interior (Ti medida y Ti simulada) y exterior (Text), (a) ambiente sur, y (b) ambiente norte, para la configuración de medida 12 del 24 al 28 de febrero del 2017.

La actividad humana de pernoctación de tres personas (dos adultos y un adolescente), se consideró con valores de tasa metabólica (Met) como variable de ingreso a la simulación (ver Fig. 8) de $40 \mathrm{~W} / \mathrm{m}^{2}$ cada uno. Para un hombre promedio (30 años, $70 \mathrm{~kg}, 1,73 \mathrm{~m}$ de altura, 1,8 $\mathrm{m}^{2}$ de superficie) [26]. Entonces, cada persona aporta $72 \mathrm{~W}$. La operatividad humana por $5 \mathrm{~h}$ se consideró para una actividad de trabajo de oficina con un aporte de $126 \mathrm{~W}$ por hora.

Los resultados encontrados en la Fig. 7 entre lo real medido y lo simulado, se validan a partir de técnicas estadísticas que utilizan dos índices diferentes, el coeficiente de la raíz del error cuadrático de la media o CV (RSME) ecuación (1), que permite determinar lo cerca que un modelo se ajusta a los datos medidos; y el error de sesgo medio (MBE) ecuación (2), que mide que tan cerca los datos simulados por el modelo, corresponden a los datos reales medidos [27]. Empleando estos índices se generaron resultados de error porcentual para la condición ambiental de los modelos.

\begin{tabular}{|c|c|c|c|}
\hline Field & Units & Obji & Oble \\
\hline Name & & Pernoctacion Sur & Penociacion Notie \\
\hline Zone or Zonel ist Name & & Domitoio_Sur & Domitorio_Nate \\
\hline Number of People Schedule Name & & Ocupacion Pers & Ocupacion Pers \\
\hline Number of People Calculation Nethod & & People & People \\
\hline Number of People & & 0 & 3 \\
\hline People per Zone Floor Area & personim2 & & \\
\hline Zone Floor Ärea per Person & n2/person & & \\
\hline Fraction Radiant & & 0.3 & 0.3 \\
\hline Sensible Heat Fracion & & aubocalculaie & aulocalculate \\
\hline Áctivity Level Schedule Name & & CalPemociacion & Cal Pernoctacion \\
\hline
\end{tabular}

Fig. 8. Campo de ingreso de variable para la simulación, número de personas y su actividad.

$$
\begin{aligned}
& C V(R M S E)=\frac{\sqrt{\sum_{i=0}^{N_{i}} \frac{\left(M_{i}-S_{i}\right)^{2}}{N_{i}}}}{\frac{1}{N_{i}} \sum_{i=1}^{N_{i}} M_{i}} \\
& M B E=\frac{\sum_{i=1}^{N_{i}}\left(M_{i}-S_{i}\right)}{\sum_{i=1}^{N_{i}} M_{i}}
\end{aligned}
$$

Donde, $\boldsymbol{M}_{\boldsymbol{i}}$ representa los datos medidos, $\boldsymbol{S}_{\boldsymbol{i}}$ los datos simulados, y $\boldsymbol{N}_{\boldsymbol{i}}$ el número de datos. Típicamente un modelo es declarado calibrado o validado si se obtiene CV (RMSE) y MBE, dentro del $30 \%$ y $\pm 10 \%$ respectivamente [28]. Para el presente estudio se obtiene CV (RMSE) $15 \%$ para el ambiente sur, y $16 \%$ para el ambiente norte; y $M B E-1 \%$ para el ambiente sur, y -2 $\%$ para el ambiente norte.

Con el modelo validado, se infiere que la representación virtual del MEV mediante software por computadora, tendrá un comportamiento tal cual se presenta en la realidad y es posible, realizar cambios sobre determinados componentes constructivos como las paredes dado a que estos, por sus valores de transmitancias (U) de llevar una capa de barro por el exterior de 0,02 $\mathrm{m}$ y 0,40 $\mathrm{m}$ de espesor de adobe es, $\mathrm{U}=1,63 \mathrm{~W} / \mathrm{m}^{2} \cdot{ }^{\circ} \mathrm{C}$ (la norma EM.110 exige $1 \mathrm{~W} / \mathrm{m}^{2} \cdot{ }^{\circ} \mathrm{C}$ ). Según la norma, no cumplen con las exigencias y deben modificarse o adicionarse mayores elementos constructivos que mejoren su aislamiento o resistencia térmica al paso del calor. Por tal razón, se simula el MEV con nuevas consideraciones de paredes tal como se muestra en la Tabla 3, donde, el punto 1 es tal cual se encuentran las paredes del MEV, el 2 lleva un recubrimiento de yeso de $0,03 \mathrm{~m}$ de espesor por el interior y exterior $\left(U=1,26 \mathrm{~W} / \mathrm{m}^{2} \cdot{ }^{\circ} \mathrm{C}\right)$, el 3 es una doble pared de adobe de 0,20 $\mathrm{m}$ de espesor con espacio de aire de 0,05 $\mathrm{m}$ y recubrimiento de barro de 0,02 $\mathrm{m}$ de espesor $\left(U=1,23 \mathrm{~W} / \mathrm{m}^{2} \cdot{ }^{\circ} \mathrm{C}\right)$, el 4 a diferencia del 3 lleva recubrimiento de yeso exterior e interior de 0,03 $\mathrm{m}$ de espesor $\left(U=1,03 \mathrm{~W} / \mathrm{m}^{2} \cdot{ }^{\circ} \mathrm{C}\right)$, el 5 a diferencia del 4 lleva 
fibra de vidrio en vez de aire $\left(\mathrm{U}=0,49 \mathrm{~W} / \mathrm{m}^{2} \cdot{ }^{\circ} \mathrm{C}\right)$. Todos ellos con el consecuente incremento de temperatura interior respecto de 1 y representado entre paréntesis. TABLA III

Incremento de la temperatura interior (entre paréntesis) según las opciones de mejora de las paredes y referenciadas con el componente 1 .

\begin{tabular}{|c|c|c|}
\hline Componentes constructivos de paredes & $\begin{array}{l}\text { Ti S Sim } \\
\left({ }^{\circ} \mathrm{C}\right)\end{array}$ & $\begin{array}{l}\text { Ti N Sim } \\
\left({ }^{\circ} \mathrm{C}\right)\end{array}$ \\
\hline 1. Barro2 $\mathrm{cm}$-adobe $40 \mathrm{~cm}$ & 16,2 & 18,1 \\
\hline 2. Yeso3cm-adobe $40 \mathrm{~cm}$-yeso $3 \mathrm{~cm}$ & $\begin{array}{c}16,8 \\
\left(0,5^{\circ} \mathrm{C}\right)\end{array}$ & $\begin{array}{c}18,7 \\
\left(0,6^{\circ} \mathrm{C}\right)\end{array}$ \\
\hline $\begin{array}{l}\text { 3. Barrozcm-adobe } 20 \mathrm{~cm} \text {-aire } 5 \mathrm{~cm}- \\
\text { adobe } 20 \mathrm{~cm}\end{array}$ & $\begin{array}{c}16,9 \\
\left(0,7^{\circ} \mathrm{C}\right)\end{array}$ & $\begin{array}{c}18,9 \\
\left(0,8^{\circ} \mathrm{C}\right)\end{array}$ \\
\hline $\begin{array}{l}\text { 4. Yeso } 3 \mathrm{~cm} \text {-adobe } 20 \mathrm{~cm} \text {-aire } 5 \mathrm{~cm} \text { - } \\
\text { adobe } 20 \mathrm{~cm} \text {-yeso } 3 \mathrm{~cm}\end{array}$ & $\begin{array}{c}17,2 \\
\left(0,9^{\circ} \mathrm{C}\right)\end{array}$ & $\begin{array}{c}19,2 \\
\left(1,1^{\circ} \mathrm{C}\right)\end{array}$ \\
\hline $\begin{array}{l}\text { 5. Yeso } 3 \mathrm{~cm} \text {-adobe } 20 \mathrm{~cm} \text {-fibra vidrio } 5 \mathrm{~cm} \text { - } \\
\text { adobe } 20 \mathrm{~cm} \text {-yeso } 3 \mathrm{~cm}\end{array}$ & $\begin{array}{c}19,1 \\
\left(2,9^{\circ} \mathrm{C}\right)\end{array}$ & $\begin{array}{c}21,4 \\
\left(3,3^{\circ} \mathrm{C}\right)\end{array}$ \\
\hline
\end{tabular}

Nota: Las componentes constructivas del punto 2 al 5 representan propuestas constructivas de incremento de la temperatura interior, considerando el punto 2 hay un incremento promedio de $0,55^{\circ} \mathrm{C}$, y con el punto 5 de $3,1^{\circ} \mathrm{C}$.

De la tabla III la propuesta 5 respecto de 1 es la que presenta el mayor incremento de la temperatura del aire interior en los ambientes del MEV con alrededor de $3{ }^{\circ} \mathrm{C}$. Si bien no es común este tipo de componente constructivo de doble pared con aislante, es una alternativa que debería probarse y medirse para verificar y corroborar los resultados simulados.

\section{CONCLUSIONES}

- Se logró en promedio incrementos de temperatura de hasta $9,5{ }^{\circ} \mathrm{C}$ en el ambiente norte con la configuración de medida 12 , y $9,2{ }^{\circ} \mathrm{C}$ en el ambiente sur con la configuración de medida 9, ambas configuraciones consideran el manejo de las contraventanas, uso de cocina a gas, uso de sistemas de calefacción, y pernoctación. Lograr una temperatura interior cercana a la temperatura de confort de $16,8^{\circ} \mathrm{C}$ según modelo adaptativo, se alcanza con las configuraciones de medida 5 y 6 básicamente, a partir del control de contraventas y sistemas de calefacción.

- La validación de la simulación a partir de modelos de cálculos estadísticos como los índices CV (RMSE) y MBE, presentan buenos acuerdos entre la data experimental y simulada, y a partir de ello, proponer una doble pared de adobe con separación de $0,05 \mathrm{~m}$ de fibra de vidrio $\mathrm{y}$ recubrimiento exterior e interior con yeso, incrementaría la temperatura interior en $3^{\circ} \mathrm{C}$.

- La operatividad y manejo concienzudo de los elementos de una vivienda, juega un rol importante más allá que las implementaciones realizadas en ella. Sin un control adecuado de las infiltraciones que puedan existir en una vivienda ya sea por los vanos de puertas y/o ventanas, no será posible alcanzar las mejoras de los incrementos de temperatura deseadas para la comodidad térmica $y$, por el contrario, se alcanzará incomodidad térmica. Es por ello que la transferencia de conocimientos y sensibilización a las poblaciones toma un rol importante en temas como, uso y manejo de los elementos constructivos de una vivienda, causas y consecuencias que ocasionan en la salud las bajas temperaturas o "heladas", daños a la infraestructura, consecuencias en la educación respecto al rendimiento del alumnado, aprovechamiento del recurso solar, y la revaloración en el uso de materiales tradicionales y sus técnicas constructivas ancestrales.

\section{AGRADECIMIENTOS}

Este trabajo se ha realizado por la financiación recibida por el Consejo Nacional de Ciencia, Tecnología e Innovación (CONCYTEC) a través del convenio 0242018-FONDECYT. J.M agradece la beca de doctorado recibida por medio del contrato 05-2018 FONDECYT/BM.

\section{REFERENCIAS}

[1] SENAMHI. (2016, Ago. 10). Avisos SENAMH [Online]. Available: http://www.senamhi.gob.pe/_0142.php?tip_alert=022\&anio=2016\&co $\mathrm{d}=066$ (accessed Aug. 12, 2016).

[2] Presidencia del Consejo de Ministros, Plan multisectorial ante heladas y friajes, Abr., 2016.

[3] El Comercio (2016, Jul. 30). Amplían emergencia en 14 regiones por heladas y friaje, El Comercio [Online]. Available: http://elcomercio.pe/sociedad/peru/amplian-emergencia-14-regionesheladas-y-friaje-noticia-1920620 (accessed Aug. 12, 2016).

[4] M. Horn, El estado actual de las energías renovables no convencionales en el Perú, 2006. [Online]. Available: http://fc.uni.edu.pe/mhorn/ER\%20in\%2OPeru.htm (accessed Aug. 4, 2016).

[5] SENAMHI, Atlas Solar - Proyecto PER/98/G31: Electrificación rural a base de energía fotovoltaica en el Perú, Lima, 2003.

[6] G. A. Abanto, M. Karkri, G. Lefebvre, M. Horn, J. L. Solis and M. M. Gómez, "Thermal properties of adobe employed in Peruvian rural areas: Experimental results and numerical simulation of a traditional bio-composite material," Case Studies in Construction Materials, vol. 6, pp. 177-191, 2017, doi: 10.1016/j.cscm.2017.02.001

[7] V. S. K. V. Harish and A. Kumar, "A review on modeling and simulation of building energy systems," Renewable and Sustainable Energy Reviews, vol. 56, pp. 1272-1292, Apr. 2016, doi: 10.1016/j.rser.2015.12.040

[8] G. Saavedra, "Diseño, construcción y evaluación térmica de un módulo de vivienda rural en la localidad de Vilcallamas Arriba, distrito de Pisacoma, provincia Chucuito, región Puno," Tesis de Titulación, Universidad Nacional de Ingeniería, Lima - Perú, 2014.

[9] J. R. Molina, "Evaluación bioclimática de una vivienda rural alto andina de la Comunidad San Francisco de Raymina de Ayacucho," Tesis de Maestría, Universidad Nacional de Ingeniería, Lima - Perú, 2016.

[10] F. Huaylla, "Evaluación experimental de cambios constructivos para lograr confort térmico en una vivienda altoandina del Perú," Tesis de Titulación, Universidad Nacional de Ingeniería, Lima - Perú, 2010.

[11] M. Gómez, R. Espinoza and M. Horn, Energy for unserved populations, in Guide towards a sustainable energy future for the Americas, IANAS, Editor, 2016.

[12] GIZ-MVCS, Abrigando hogares - experiencias con medidas de confort térmico en viviendas rurales altoandinas, Lima, 2015. 
[13] MVCS, "Norma EM.110 Confort Térmico y Lumínico con Eficiencia Energética," Reglamento Nacional de Edificaciones, 2014. [Online]. Available: http://www.construccion.org.pe/normas/rne2012/rne2006.htm (accessed 2016, Jul. 25).

[14] N.G. Reus y J.D. Czajkowski, "Comparación entre las normas de desempeño térmico edilício de Argentina y Brasil," Ambiente Construído, vol. 16, no. 1, pp. 105-122, Jan. 2016, doi: 10.1590/s167886212016000100063.

[15] J. Francoiz y A. Guinebault, Calefacción Solar para Regiones Frías: guía tecnológica de aplicación para la vivienda y la agricultura en países en desarrollo, vol. 2. ITDG-Perú: Soluciones Prácticas, 1997.

[16] L. Harman, Confort Térmico en Viviendas Altoandinas: un enfoque integral, Care Peru, 2010.

[17] F. M. Agugliaro, F. G. Montoya, A. S. Ortega, and A. G. Cruz, "Review of bioclimatic architecture strategies for achieving thermal comfort," Renewable and Sustainable Energy Reviews, vol. 49, pp. 736-755, 2015.

[18] A. Martínez-Molina, I. Tort-Ausina, S. Cho, and J.-L. Vivancos, "Energy efficiency and thermal comfort in historic buildings: A review," Renewable and Sustainable Energy Reviews, vol. 61, pp. 7085, Aug. 2016, doi: 10.1016/j.rser.2016.03.018

[19] R. D. L. Vollaro et al., "An Integrated Approach for an Historical Buildings Energy Analysis in a Smart Cities Perspective," Energy Procedia, vol. 45, pp. 372-378, Jan. 2014, doi: 10.1016/j.egypro.2014.01.040

[20] U.S. Department of Energy's, EnergyPlus. [Online]. Available: https://energyplus.net/ (accessed 2018, Mar. 8).

[21] R. Espinoza, "Evaluación experimental de la performance de dos componentes bioclimáticas de calentamiento aplicadas en un módulo de vivienda de San Francisco de Raymina - Ayacucho con el propósito de validarlas como técnicas de estrategias bioclimáticas para viviendas rurales Alto Andinas", Tesis de Maestría, Universidad Nacional de Ingeniería, Lima - Perú, 2014.

[22] D. B. Crawley et al., "EnergyPlus: creating a new-generation building energy simulation program," Energy and Buildings, vol. 33, no. 4, pp. 319-331, Apr. 2001, doi: 10.1016/S0378-7788(00)00114-6

[23] J. Yang, H. Fu, and M. Qin, "Evaluation of Different Thermal Models in EnergyPlus for Calculating Moisture Effects on Building Energy Consumption in Different Climate Conditions," Procedia Engineering, vol. 121, pp. 1635-1641, Jan. 2015, doi: 10.1016/j.proeng.2015.09.194

[24] S. K. Alghoul, H. G. Rijabo, and M. E. Mashena, "Energy consumption in buildings: A correlation for the influence of window to wall ratio and window orientation in Tripoli, Libya," Journal of Building Engineering, vol. 11, pp. 82-86, May 2017, doi: 10.1016/j.jobe.2017.04.003

[25] D. Morillón, Atlas del bioclima de México. México, D.F., 2004.

[26] Y. Cengel and A. Ghajar, Heat and Mass Transfer: Fundamentals and Applications. Cap. 16. Heating and Cooling of Buildings, 5ta. New York: McGraw-Hill Interamericana, 2015.

[27] X. Liang, Y. Wang, M. Royapoor, Q. Wu, and T. Roskilly, "Comparison of building performance between Conventional House and Passive House in the UK," Energy Procedia, vol. 142, pp. 18231828, Dec. 2017, doi: 10.1016/j.egypro.2017.12.570

[28] G. R. Ruiz and C. F. Bandera, "Validation of Calibrated Energy Models: Common Errors," Energies, vol. 10, no. 10, p. 1587, Oct. 2017, doi:10.339o/en10101587

\section{(c) (1)}

Los artículos publicados por TECNIA pueden ser compartidos a través de la licencia Creative Commons: CC BY 4.0. Permisos lejos de este alcance pueden ser consultados a través del correo revistas@uni.edu.pe 